\title{
Method Realizing both Protocol Performance Evaluation and Validation Based on Petri Net
}

\author{
SHU De Qin ${ }^{a}$, FAN Hao ${ }^{b}$ and Zhang Liang ${ }^{c}$ \\ College of Information Science and Engineering, Shandong Agricultural University,Tan'an, \\ China

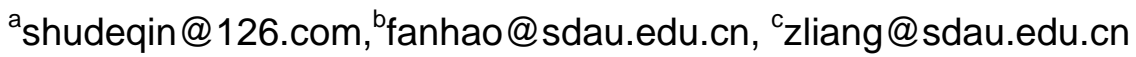 \\ Corresponding author: Fan Hao, fanhao@sdau.edu.cn.
}

Keywords: Protocol performance evaluation; Protocol validation; Petri net; Timed Petri net; QoS

\begin{abstract}
Due to its concise, simple and unambiguous qualities, the Petri net method has become a reliable and accurate way of analyzing protocol. However, with one model conducting one point, the required models of the key points of protocol analysis, namely, the protocol performance evaluation and the protocol validation, are quite different. A method of using original Petri net as a model of protocol verification is proposed to solve this problem. And without changing any part of the structure of the original Petri net, it has realized protocol performance evaluation by attaching time delay to transition, hence avoiding repeat modeling. By so doing, one model can evaluate the performance of the protocol and validate it at the same time. As an example, the paper validates $0-1$ stop and wait for protocol in detail and then evaluates the performance of the protocol by translating original Petri net of 0-1 stop and wait for protocol into timed Petri net.
\end{abstract}

\section{Introduction}

The protocol validation aims to the detection and correction of the protocol errors and defects before its realization $[1,2]$. While the protocol performance evaluation mainly aims to evaluate the throughput, data transmission delay as well as other indexes of the protocol, which is of vital importance to the qualities of service(QoS) of the protocol[3].

The Petri Net method is a reliable and accurate way of analyzing protocol [4], based on which many types of research have acquired some achievements regarding the protocol performance evaluation and the protocol validation. For instance, viewing from the perspective of protocol validation, in reference [5], the author has made Petri net model of SIP protocol and made consistency analysis to the SIP protocol in mobile networks. And in reference [6], the author has proposed a safety analysis method by colored Petri net of clock synchronization protocol, making a reachability analysis to the unsafe state by way of state equations. In reference [7], the author has established the stochastic Petri Net model of wireless sensor interface protocol WTIM and has analyzed the effect of structure parameters upon the rate of model data dropout as well as other performances by using the Markov chain. And in reference[8], the author has made full use of stochastic Petri net to evaluate the service performance quality of VoIP.

Though many results regarding the Petri net analysis method of protocols are already fruitful, there are still conflicts existing between the protocol validation and its performance evaluation. On one hand, the protocol validation model can not make an instant quantitative analysis of the protocol performance; On the other, the performance evaluation model can not reflect the various static characteristics and dynamic behaviors of the protocol. Based on the above analysis, this paper proposes a method realizing both protocol performance evaluation and protocol validation based on Petri net. It first proposes to establish original Petri net model of the protocol, finishing the protocol validation and then to fulfill protocol performance evaluation by attaching time delay to transition, without changing any part of the model structure. As an example, the paper provides the original Petri net model and timed Petri net model to 0-1 stop and wait for protocol, thus making protocol validation and its performance evaluation regarding 0-1 stop and wait for protocol. 


\section{The Basic Conception of the Original Petri Net and Timed Petri Net}

Definition $1^{[9]}$ The original Petri net can be defined as $\Sigma=(P, T, F, M)$, where

1) $P \cup T \neq \phi$

2) $P \cap T=\phi$

3) $F \subseteq((P \times T) \cup(T \times P))$

4) $\operatorname{dom}(F) \cup \operatorname{cod}(F)=P \cup T$

5) $M: P \rightarrow\{0,1,2, \cdots\}$

$\mathrm{P}$ and $\mathrm{T}$ are two sets without intersection; they are called basic elements of original Petri net. The elements in $\mathrm{P}$ are called $\mathrm{P}$ - element or places. The elements in $\mathrm{T}$ are called T- element or transitions. $\mathrm{F}$ is called flow relation; $\operatorname{dom}(F)$ and $\operatorname{cod}(F)$ are called the former domain and the after domain. $\mathrm{M}$ is the marking of $\Sigma$.

Definition $2^{[9]}$ The original Petri net $\Sigma=(P, T, F, M)$ consists of the following transition rules.

1) To $t \in T$, if $\forall p \in^{\circ} t: M(p) \geq 1$, called $M$ authorizes $t$ to fire, or $t$ be called enabled, marked as $M[t>$.

2) If the marking $M$ authorizes $t$ to fire, transition $t$ can fire under $M, \quad t$ fires under $M$, resulting in new marking $M^{\prime}$ (marked as $M\left[t>M^{\prime}\right)$, to $\forall p \in P$ :

$$
M^{\prime}(p)=\left\{\begin{array}{l}
M(p)-1 \text { when } p \in{ }^{\bullet} t-t^{\cdot} \\
M(p)+1 \text { when } p \in t^{\bullet}-{ }^{\cdot} t \\
M(p) \text { others }
\end{array}\right.
$$

Definition $3^{[9]}$ Let $\Sigma=(P, T, F, M)$ be a Petri net, $P=\left\{p_{1}, p_{2}, \cdots, p_{m}\right\}, \quad T=\left\{t_{1}, t_{2}, \cdots, t_{n}\right\}$, the structures $(P, T ; F)$ of Petri net $\sum$ can be expressed by a matrices with $\mathrm{n}$ rows m columns $A=\left[a_{i j}\right]_{n \times m}$, where $a_{i j}=a_{i j}^{+}-a_{i j}^{-} \quad i \in\{1,2, \cdots, n\}, \quad j \in\{1,2, \cdots, m\}$.

$$
\begin{aligned}
a^{+} & =\left\{\begin{array}{ll}
1 & \text { if } \quad\left(t_{i}, s_{j}\right) \in F \\
0 & \text { else }
\end{array} \quad i \in\{1,2, \cdots, n\}, j \in\{1,2 \cdots, m\}\right. \\
a_{i j}^{-} & = \begin{cases}1 & \text { if } \quad\left(p_{i}, t_{j}\right) \in F \\
0 & \text { else }\end{cases}
\end{aligned}
$$

$A$ is called the incidence matrix of $\Sigma$ (or net $N=(P, T ; F)$ ).

Definition $4^{[9]}$ Let $\Sigma=\left(P, T, F, M_{0}\right)$ be a Petri net, $s \in S$, suppose there exists a positive integer $B$, such that $\forall M \in R\left(M_{0}\right): M(s) \leq B$, then the place $s$ is called bounded, and the smallest positive integer $B$, which meets this condition, is called the bound of place $s$, marked as $B(s)$. That is, $B(s)=\min \left\{B \mid \forall M \in R\left(M_{0}\right): M(s) \leq B\right\}$, when $B(s)=1$, the place $s$ is called safe.

Definition $5^{[9]}$ Let $\Sigma=\left(P, T, F, M_{0}\right)$ be a Petri net, $t \in T$. Suppose for $\forall M \in R\left(M_{0}\right)$, there exists $\sigma \in T^{*}$, such that $M\left[\sigma>M^{\prime}\right.$ and $M^{\prime}[t>$, then $t$ is live. If each transition in a Petri net is alive, then the whole net is alive.

Definition $6^{[9]}$ Let $N=(P, T ; F)$ be a Petri net, $P|=m, \quad T|=n$, A is the incidence matrix of $N$.

1) If nontrivial m-dimension nonnegative integer vector $Y$, that is satisfied $A Y=0$, then $Y$ is called an $P$-invariant of the net $N$.

2) If nontrivial n-dimension nonnegative integer vector $X$, that is satisfied $A^{T} X=0$, then $X$ is called an $T$ - invariant of the net $N$.

Definition $7^{[9]}$ Timed Petri net is a set with five elements: $T P N=(P, T, F, \tau, M)$, where $P, T, F$ have the same meaning with $P, T, F$ in definition 1. $\tau$ is time mapping function $\tau \rightarrow 0 \cup Q^{+}$( $Q^{+}$is positive rational number ), it defines duration time of each transition. If the duration time is 0 , it is called instantaneous transition, otherwise (duration time is not 0 ), it is called timed transition. $M: P \rightarrow\{0,1,2, \cdots\}$ is the net marking. 
Please refer to reference [9] for other conceptions and theorems regarding Petri net.

\section{A Petri-net-based Method for Protocol Validation and Protocol Performance Evaluation}

\section{Protocol Validation}

Many specialties of the Petri net can find its actual physical meaning in the protocol. The following Table 1 briefly listed the corresponding relations between them two.

(1) Static analysis

The static analysis mainly refers to the analysis of the structural characteristics of the protocol: structure-activity, boundedness, conservativeness, security, P-invariants, T-invariants, etc. For instance, P-invariants mean that the resources (data frame, response frame) of the protocols are safe. By analyzing the topology features of the Petri net, we can analyze many properties of the protocol and hence verify the protocol's validity.

(2) Dynamic analysis

It is to analyze the dynamic features of the protocol, such as action sequences, reachability, liveness, etc. The reachability graph of Petri net, consisting of some information useful for the protocol validity, can analyze the accessibility (from one state to another), action sequences, the recoverability of the protocol error state, etc.

Table 1 the contents of protocol validity and their according Petri net analyzing methods

\begin{tabular}{|l|l|}
\hline \multicolumn{1}{|c|}{ the contents of protocol validity } & \multicolumn{1}{c|}{ Petri net analyzing property } \\
\hline Accessibility among protocol state & reachability analysis \\
\hline deadlock & $\begin{array}{l}\text { The liveness, deadlock and trap theory of Petri } \\
\text { net }\end{array}$ \\
\hline $\begin{array}{l}\text { Whether some parameters(channels, buffers)in } \\
\text { the protocol are bounded }\end{array}$ & boundedness \\
\hline $\begin{array}{l}\text { Whether some parameters are safe in the course } \\
\text { of the running of the protocol }\end{array}$ & P-invariants \\
\hline $\begin{array}{l}\text { Whether the action sequences of the protocol are } \\
\text { sustainable }\end{array}$ & T-invariants \\
\hline $\begin{array}{l}\text { Whether the action sequences of the protocol are } \\
\text { legal }\end{array}$ & Petri net transition fire sequences \\
\hline
\end{tabular}

2. Protocol Performance Evaluation

The efficiency and throughput of the protocol, data transmission delay, those are also of vital importance in practical application, which can be fully analyzed by the timed Petri net. The delayed time for the transition definition of the original Petri net can help to realize the protocol performance evaluation, which is also the advantage of this paper. Two points need to be considered when evaluating the protocol performance.

(1) The maximum throughput

The maximum data frames successfully sent out every second is the maximum throughput of the data link, marked as $\lambda_{\max }$.

(2) The average propagation latency

The average time needed for the correctly sent out of the data frames, marked as $t_{A V}$.

\section{A practical example: The protocol validation and performance evaluation for 0-1 stops and wait for protocol}

The resend of the wrong data frames from the transmit port of the 0-1 stops and wait for protocol is automatic, hence the protocol applying this kind of error control system is called ARQ (Automatic Repeat request), the detailed description of which see reference [10].

1. The original Petri net model of 0-1 stops and wait for protocol

The following fig. 1 is the Petri net model of 0-1 stops and wait for protocol, with the description 
of symbols of fig. 1 in Table 2.

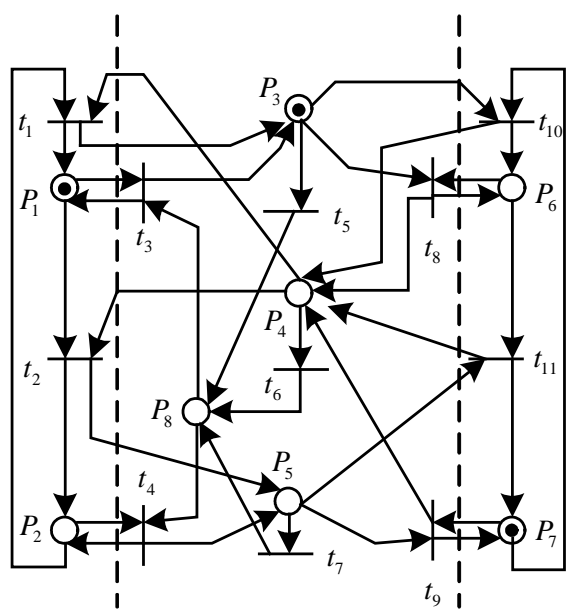

the state of the sender the state of the channel the state of the receiver

Fig. 1 the Petri net model of 0-1stops and wait

Table 2 the description of symbols of $0-1$ protocol's Petri net model

\begin{tabular}{|l|l|l|l|l|l|}
\hline $\begin{array}{l}\text { Symbols } \\
\text { name }\end{array}$ & $\begin{array}{l}\text { Place/Transition } \\
\text { Significance }\end{array}$ & $\begin{array}{l}\text { Symbols } \\
\text { name }\end{array}$ & $\begin{array}{l}\text { Place/Transition } \\
\text { Significance }\end{array}$ & $\begin{array}{l}\text { Symbols } \\
\text { name }\end{array}$ & $\begin{array}{l}\text { Place/Transition } \\
\text { Significance }\end{array}$ \\
\hline $\mathrm{P}_{1}$ & Wait ACK & $\mathrm{P}_{8}$ & $\begin{array}{l}\text { Lose ACK or data } \\
\text { frame }\end{array}$ & $\mathrm{t}_{7}$ & Lose [1] frame \\
\hline $\mathrm{P}_{2}$ & Wait ACK & $\mathrm{t}_{1}$ & $\begin{array}{l}\text { Receive ACK, } \\
\text { send [0] frame }\end{array}$ & $\mathrm{t}_{8}$ & $\begin{array}{l}\text { Receive duplicate [0] } \\
\text { frame, do not send host }\end{array}$ \\
\hline $\mathrm{P}_{3}$ & $\begin{array}{l}{[0] \text { frame in the }} \\
\text { channel }\end{array}$ & $\mathrm{t}_{2}$ & $\begin{array}{l}\text { Receive ACK, } \\
\text { send [1] frame }\end{array}$ & $\mathrm{t}_{9}$ & $\begin{array}{l}\text { Receive duplicate [1] } \\
\text { frame, do not send host }\end{array}$ \\
\hline $\mathrm{P}_{4}$ & $\begin{array}{l}\text { ACK in the } \\
\text { channel }\end{array}$ & $\mathrm{t}_{3}$ & $\begin{array}{l}\text { [0] frame timeout } \\
\text { retransmission }\end{array}$ & $\mathrm{t}_{10}$ & $\begin{array}{l}\text { Receive [0] frame, } \\
\text { send host }\end{array}$ \\
\hline $\mathrm{P}_{5}$ & $\begin{array}{l}\text { [1]frame in the } \\
\text { channel }\end{array}$ & $\mathrm{t}_{4}$ & $\begin{array}{l}\text { [1] frame timeout } \\
\text { retransmission }\end{array}$ & $\mathrm{t}_{11}$ & $\begin{array}{l}\text { Receive [1] frame, } \\
\text { send host }\end{array}$ \\
\hline $\mathrm{P}_{6}$ & $\begin{array}{l}\text { Expected } \\
\text { receive [1]frame }\end{array}$ & $\mathrm{t}_{5}$ & Lose [0] frame & & \\
\hline $\mathrm{P}_{7}$ & $\begin{array}{l}\text { Expected } \\
\text { receive [0]frame }\end{array}$ & $\mathrm{t}_{6}$ & Lose ACK frame & & \\
\hline
\end{tabular}

In fig.1, P8 represents information loss, which contains: a.[0] frame loss, b.[1] frame loss, c. ACK loss. In fig.1, all of three of them are represented by place P8 with no distinction, conducting no effect on the analysis of protocols.

In fig. 1 , the initial marking of Petri net is: $M_{0}=[1,0,1,0,0,0,1,0]$, which means that the protocol is in this state: the sender host has already sent out the [0]data frames of $\mathrm{N}(\mathrm{S})=0$, waiting for the ACK signal from the receiver. The channel is in the state of sending [0] data frame. While the receiver host is in the state of expecting the [0] data frame. Without loss of generality, the marking can be considered as the initial state of the protocol.

2. The static analysis of the 0-1 stops and wait for protocol

(1)The verification of protocol conservativeness by P-invariants

We consider the process of the sending and receiving of data frames(including the sending and receiving of ACK) instantaneous. If the protocol is right, the sender host would be in such state: sent the [0]frame wait for ACK or having sent the [1] frame wait for ACK. The receiver host is in such state: expecting the receiving of [0] frame or expecting the receiving of [1] frame. The channel is in such state: in the course of sending [0] frame or in the course of sending ACK or information 
loss. Corresponding to the Petri net model in fig.1, if the protocol meets the conditions, $\left\{p_{1}, p_{2}\right\}$ can express the state of the sender. The state of the receiver is then described as $\left\{p_{6}, p_{7}\right\}$, the state of the channel is then described as $\left\{p_{3}, p_{4}, p_{5}, p_{8}\right\}$, all of which being the support sets of the P-invariants.

Fig. 2 shows the solution of the equation $A Y=0$. According to the solution, $0-1$ wait protocol can be well verified by P-invariants.

$$
A=\left[\begin{array}{cccccccc}
1 & -1 & 1 & -1 & 0 & 0 & 0 & 0 \\
-1 & 1 & 0 & -1 & 1 & 0 & 0 & 0 \\
0 & 0 & 1 & 0 & 0 & 0 & 0 & -1 \\
0 & 0 & 0 & 0 & 1 & 0 & 0 & -1 \\
0 & 0 & -1 & 0 & 0 & 0 & 0 & 1 \\
0 & 0 & 0 & -1 & 0 & 0 & 0 & 1 \\
0 & 0 & 0 & 0 & -1 & 0 & 0 & 1 \\
0 & 0 & -1 & 1 & 0 & 0 & 0 & 0 \\
0 & 0 & 0 & 1 & -1 & 0 & 0 & 0 \\
0 & 0 & -1 & 1 & 0 & 1 & -1 & 0 \\
0 & 0 & 0 & 1 & -1 & -1 & 1 & 0
\end{array}\right] \quad\left[\begin{array}{c}
Y_{1} \\
Y_{2} \\
Y_{3} \\
Y_{4} \\
Y_{5} \\
Y_{6} \\
Y_{7} \\
Y_{8}
\end{array}\right]=c_{1}\left[\begin{array}{c}
1 \\
1 \\
0 \\
0 \\
0 \\
0 \\
0 \\
0
\end{array}\right]+c_{2}\left[\begin{array}{l}
0 \\
0 \\
0 \\
0 \\
1 \\
1 \\
0
\end{array}\right]+c_{3}\left[\begin{array}{l}
0 \\
0 \\
1 \\
1 \\
1 \\
0 \\
0 \\
1
\end{array}\right]
$$

Fig.2 incidence matrix A and solution of equation $\mathrm{AY}=0$

(2) the verification of protocol sustainability by T-invariants

We can see from the equation $A^{T} X=0$ that $\left\{t_{5}, t_{3}\right\},\left\{t_{6}, t_{3}, t_{8}\right\},\left\{t_{10}, t_{2}, t_{11}, t_{1}\right\}$ are all support sets of T-invariants. The T-invariants reflects the cycle subsystem of Petri net model. Hence the $\left\{t_{5}, t_{3}\right\}$ invariant support set reflects the following practical meaning: [0] data frame gets lost in the channel, and after waiting for ACK for a while, the sender would resend [0]frame automatically due to timer expire. $\left\{t_{6}, t_{3}, t_{8}\right\} \quad$ Invariant support set reflects the recovery ability of the system after the loss of ACK. While $\left\{t_{10}, t_{2}, t_{11}, t_{1}\right\}$ support set reflects that the system can successfully loop the execution of the following action sequences: sending[0]frame, receiving [0]frame and sending ACK, sending[1]frame, receiving [1]frame and sending ACK. There are also other support sets of T-invariants, which we would not discuss here. The verification of T-invariants indicates that $0-1$ wait protocol has the ability of recovery ability in an error state and the ability to continuously send data frames.

3.The dynamic analysis of 0-1 stop and wait for protocol

The reachability graph of the Petri model in fig. 1 is introduced, which can conduct the following dynamic analysis and verification to the $0-1$ stop and wait for protocol.

(1)the verification of the protocol sending, receiving and channel boundedness

In 0-1 stop and wait for protocol, the data frames use serial number [0]/[1] alternatively, hence the capacity of the send and receive buffers, as well as the channel, should be 1 . After the observation of fig.3, we can know that when the original marking $M_{0}=[1,0,1,0,0,0,1,0]$ is given, for $\forall s \in S, \forall M \in R\left(M_{0}\right): M(s) \leq 1$. The Petri net in fig. 1 is then safeness, verifying the fact that the capacity of the send and receive buffers as well as the channel is 1.

(2)the verification of protocol deadlock-free by the liveness of Petri net

According to fig.3, each transition of the Petri net is alive, namely, the net in the figure is alive, indicating that there is not deadlock in $0-1$ stop and wait for protocol. 


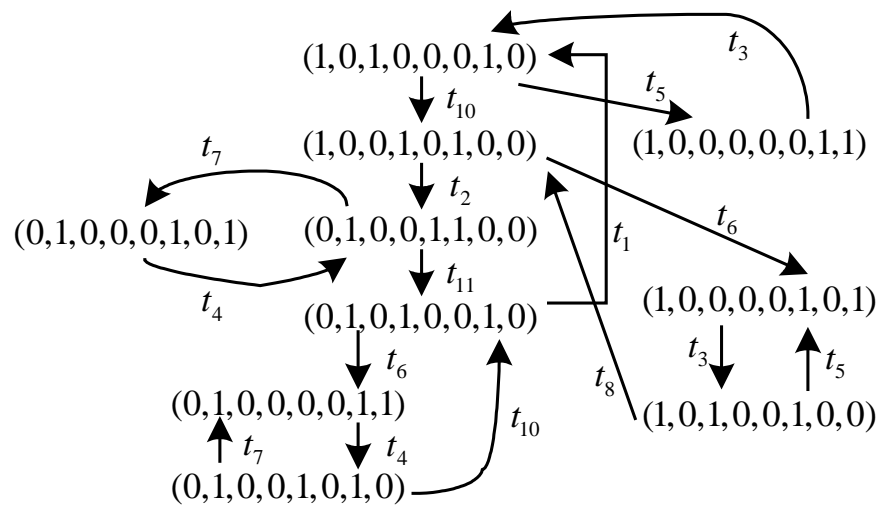

Fig.3 the reachability graph of Petri net

\section{The performance evaluations of 0-1stop and wait for protocol}

This paper adopts timed Petri net to evaluate the protocol performance of $0-1$ stop and wait for protocol, adding the following latency, (see Table 3), to the transitions of the models in fig.1, the result timed Petri net marked as $\Sigma_{2}$.

Table 3 the latency time of the transition of $\Sigma_{2}$

\begin{tabular}{|c|c|c|c|c|c|}
\hline $\begin{array}{l}\text { transition } \\
\text { name }\end{array}$ & latency & $\begin{array}{l}\text { Transition } \\
\text { name }\end{array}$ & latency & $\begin{array}{l}\text { transition } \\
\text { name }\end{array}$ & latency \\
\hline$t_{1}$ & $\tau_{1}=t_{f}+t_{p r}+t_{p}$ & $t_{5}$ & $\tau_{5}=0$ & $t_{9}$ & $\tau_{9}=t_{p}+t_{p r}+t_{a}$ \\
\hline$t_{2}$ & $\tau_{2}=t_{f}+t_{p r}+t_{p}$ & $t_{6}$ & $\tau_{6}=0$ & $t_{10}$ & $\tau_{10}=t_{p}+t_{p r}+t_{a}$ \\
\hline$t_{3}$ & $\tau_{3}=t_{\text {out }}$ & $t_{7}$ & $\tau_{7}=0$ & $t_{11}$ & $\tau_{11}=t_{p}+t_{p r}+t_{a}$ \\
\hline$t_{4}$ & $\tau_{4}=t_{\text {out }}$ & $t_{8}$ & $\tau_{8}=t_{p}+t_{p r}+t_{a}$ & & \\
\hline
\end{tabular}

Explanation of the latency:

$t_{f}:$ The transmission delay of the data frames

$t_{a}:$ The transmission delay of response signal

$t_{p}$ : The propagation delay resulting from the fact that the data frames and ACK propagate along the link

$t_{p r}$ : The processing time after the receiving of data frames and ACK

$t_{\text {out }}:$ The setup time of the expiry timer

The meaning of transition $t_{1}$ is to receive ACK and send [0] frame. The latency is then defined as $\tau_{1}=t_{f}+t_{p r}+t_{p}$.It is the same with the latency of transition $t_{2} 、 t_{8} 、 t_{9} 、 t_{10} 、 t_{11}$. While the meanings of $t_{5} 、 t_{6} 、 t_{7}$ are all signal loss, the process o $\mathrm{f}$ that can be viewed as instantaneous, with its latency 0 .However, the fact is that not all the signals lose. The probability of its happening is defined as $p$.We are to make the following evaluations for the $0-1$ stop and wait for protocol:

(1) the confirmation of the retransmission time-- $t_{\text {out }}$ of the timer

The decision of the retransmission time of the timer should be carefully made. According to fig.3, the action sequence of "from the transmission of data frames to the receiving of response frame" is as follows. $M_{0}\left[t_{10}>M_{1}\left[t_{2}>M_{2}\right.\right.$

where: $\quad M_{1}=[1,0,0,1,0,1,0,0] \quad M_{2}=[0,1,0,0,1,1,0,0]$

$\tau_{10}=t_{p}+t_{p r}+t_{a} \quad \tau_{2}=t_{f}+t_{p r}+t_{p}$

According to the action sequence $M_{0}\left[t_{10}>M_{1}\left[t_{2}>M_{2}\right.\right.$, we can get fig.4. We can know from fig. 4 that $t_{\text {out }}>t_{p}+t_{p r}+t_{a}+t_{p}+t_{p r}$. Generally speaking, to the ACK frame transmission time $t_{a}$ and processing time $t_{p r}$, which much less than the propagation latency $t_{p}$. There transmission time can be defined as more than twice of the retransmission time. For the convenience of discussion, we take twice of the retransmission time, namely, $t_{\text {out }}=2 t_{p}$. 
(2) the average propagation latency

According to the action sequence $M_{0}\left[>t_{10} M_{1}\left[>t_{2} M_{2}\right.\right.$, the least time interval $t_{T}$ between two successfully sent data frames is:

$t_{T}=\tau_{10}+\tau_{2}=t_{p}+t_{p r}+t_{a}+t_{p r}+t_{p}+t_{f}=t_{f}+t_{\text {out }}=t_{f}+2 t_{p}$. With the probability of happening of $t_{5}, t_{6}, t_{7}$ being $p$ and the allowed retransmission times being unlimited, we can know that the average time $t_{A V}$ for the correctly of a data frame is:

$$
t_{A V}=t_{T}+(1-p) \sum_{i=1}^{\infty} i p^{i} t_{T}=t_{T} /(1-p)
$$

(3) the maximum throughput

The successfully sent off the maximum number of data frame each second is the maximum throughput of the link, marked as $\lambda_{\max }$. Apparently, $\lambda_{\max }=1 / t_{A V}=(1-p) / t_{T}$.

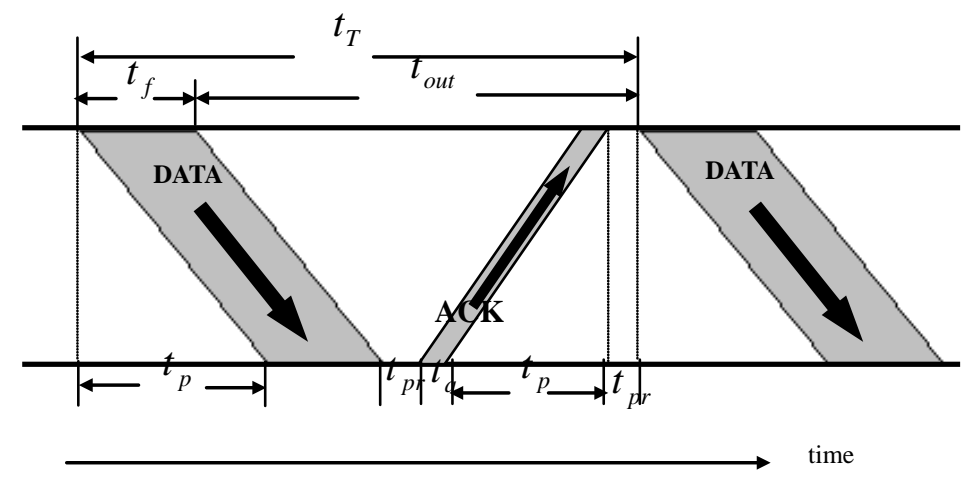

Fig.4 the time relation in stop and wait for protocol

\section{Conclusion}

Compared with the existing references, the main feature of this paper is to establish the protocol model by original Petri net and to verify the protocol. The time factor is then added to the original Petri net and transformed into timed Petri net, and then we can do the protocol performance evaluation. In this way, when it comes to protocol verification, the existing theories regarding Petri net can be taken well advantage of. When it comes to the protocol performance, it can use Petri net of protocol verification without changing the model. This paper offers a method of protocol verification with original Petri net by providing the case of $0-1$ stop and wait for protocol, to which we have made relative complete static and dynamic formal verification. By giving latency time to the transition of original Petri net, we have transformed the original Petri net into timed Petri net and then strictly conducted performance analysis with it, which is one of the main advantages of this paper. And in our later study, we will further study the semantic verification of the protocol, exploiting the language and semantics of Petri net.

\section{Acknowledgement}

This work is supported by Science and Technology project of colleges and universities in Shandong Province and Youth Science(J11LG26) and Youth Technology Innovation Fund Project of Shandong Agricultural University (23659).

\section{References}

[1]Xu Jun, Zhang Xiaoyu, Wang Xiuyan, 3GPP Mobile Communication System Standard MAC Layer Design Based on ARM+DSP[J], Computer Measurement \& Control. 2015,23(1):250-253.

[2]LI Kai, Ma Hui-chao, Research on unknown label detection protocol based on RFID[J], 
Application Research of Computeers. 2015,32(3):814-820.

[3]Tang Ya-juan, Countermeasures on Application Level Low-Rate Denial-of-Service Attack [J], Information and Communications Security Lecture Notes in Computer Science, 2012, 7618:70-80.

[4]WU Hong-Yue , DU Yu-Yue, A Logical Petri Net-Based Approach for Web Service Cluster Composition[J], Chinese Journal of computers, 2015,38(1):204-217.

[5]Dong-min Wang, Research and Implementation of Sip Conformance Testing Suite Based on Petri Net [D], Beijing University of Posts and Telecommunications, 2011.

[6]FENG Dong-gin, SHEN Jia-jun, CHU Jian, Analysis of clock synchronization protocol security using colored Petri net[J], Control and Decision,29(12),2014,2144-2150.

[7]Wu Guo-Guang, WTIM Modeling and Dynamic Performance Evaluation based on Petri net[J], Journal of the South China University of Technology,41(9),2013,107-111.

[8]Shevtekar A, Ansari N. Do Low Rate DoS Attacks Affect QoS Sensitive VoIP Traffic[C] //Proceedings of IEEE International Conference on Communications. 2006, 2153-2158.

[9] Wu Z H. The Introductory Theory of Petri net[M]. Beijing: China, Machine Press, 2006.

[10] Wang Yong, Zhang longfei, Computer networks[M]. Beijing: China, Machine Press, 2015. 